\title{
Facteurs impliqués dans le remodelage de la chromatine au cours de la spermiogenèse
}

\author{
Sophie ROUSSEAUX, Cécile CARON, Christophe PIVOT-PAJOT, Anne Karen FAURE, \\ Mira HAZZOURI, Bernard SELE, Saadi KHOCHBIN
}

Unité INSERM U309, Université Joseph Fourier, Institut Albert Bonniot, Grenoble, France

\section{RESUME}

Les spermatides rondes sont les cellules issues de la méiose dont le noyau haploïde présente initialement une structure similaire à celle d'une cellule somatique. Au cours de la maturation post-méiotique de la spermatide, ou spermiogenèse, la chromatine subit un remodelage au cours duquel le noyau de la spermatide s'allonge puis se condense pour former le noyau spermatique, dont la chromatine présente une structure très spécifique et très compacte. Lors de ce remodelage, les histones sont remplacées par les protéines de transition puis par les protamines, protéines nucléaires spécifiques du spermatozoïde. Immédiatement avant leur remplacement, les protéines histones sont hyperacétylées. Nous avons entrepris de caractériser précisément l'acétylation des histones cœur du nucléosome au cours de la spermatogenèse murine. Nous avons ainsi montré que, chez la souris, les histones cœur H2A, H2B, H3 et $\mathrm{H} 4$ sont hyperacétylées dans les spermatides en cours d'élongation. Nous avons ensuite montré qu'une dégradation des histones déacétylases (HDACs) est responsable de cette augmentation brutale du niveau d'acétylation en début d'élongation. Enfin, chez l'homme, nos données suggèrent qu'un processus semblable est mis en jeu lors de la spermatogenèse normale. De plus, une forte déplétion en cellules germinales, telle qu'elle est observée dans les syndromes des cellules de Sertoli isolées (SCO), est associée a une augmentation très importante de l'acétylation du noyau des cellules de Sertoli. L'ensemble de ces données apporte les bases nécessaires à la compréhension des mécanismes et à l'identification de facteurs impliqués dans les différentes étapes du remodelage postméiotique de la chromatine.
Mots Clés : chromatine, spermiogenèse, spermatide, histones, acétylation

\section{INTRODUCTION}

En Biologie de la Reproduction humaine la technique d'ICSI (intracytoplasmic sperm injection), dans laquelle un spermatozoïde est directement microinjecté dans le cytoplasme de l'ovocyte, a révolutionné la prise en charge de l'infertilité masculine. Ainsi, les patients atteints d'un déficit sévère de la spermatogenèse peuvent actuellement espérer une procréation intra-conjuguale avec leurs propres gamètes. Cependant, le succès de cette technique pose non seulement la question du risque de transmission d'une anomalie génétique ou chromosomique à l'éventuelle descendance de ces hommes mais aussi celle des conséquences, notamment d'ordre épigénétique, de ces techniques sur le développement de l'embryon puis de l'individu qui en est issu.

En effet, par les techniques d'ICSI, il est devenu possible d'injecter dans les ovocytes humains des spermatozoïdes dont la structure est anormale, qu'ils soient immobiles, immatures, voire même des spermatides, lesquelles ont un contenu génétique haploïde mais un noyau dont la structure diffère de celui du spermatozoïde mature $[32,33,43$, $44,52]$. On ignore tout des conséquences, notamment épi-

\section{Correspondance :}

Dr Sophie Rousseaux - INSERM U309, Institut Albert Bonniot, Domaine de la Merci, 38706 La Tronche Cedex France $\mathrm{Tel}$ (33) 04.76.54.95.12 - Fax (33) 04.76.54.95.95 Email sophie.rousseaux@ujf-grenoble.fr 
génétiques, de telles pratiques sur le développement de l'embryon et de l'individu [48, 49].

Dans ce contexte, l'étude structurale du noyau du spermatozoïde et de ses précurseurs, les spermatides, telle qu'elle est présentée ici, est d'un grand intérêt.

\section{Dans la cellule somatique, l'unité de base de la chromatine est le nucléosome}

Les spermatides rondes sont les cellules issues de la méiose dont le noyau haploïde présente initialement une structure similaire à celle d'une cellule somatique.

Dans la cellule somatique, le nucléosome est l'unité de base de la chromatine. Deux de chacune des histones cœur, $\mathrm{H} 2 \mathrm{~A}, \mathrm{H} 2 \mathrm{~B}, \mathrm{H} 3$ et $\mathrm{H} 4$ (poids moléculaire 10-16 KDa) forment un octamère autour duquel 146 paires de bases d'ADN sont enroulées, formant le nucléosome. La portion centrale de la cinquième histone, $\mathrm{H} 1$, serait associée à l'ADN lors de son entrée et de sa sortie du nucléosome [50].

Pour tous les processus nécessitant l'accès à l'ADN, notamment la transcription ou la réplication, la structure de la chromatine doit subir un remodelage complexe et régulé. La compréhension du remodelage chromatinien représente une étape cruciale dans de nombreux domaines de la biologie et de la médecine. Deux groupes d'enzymes sont actuellement connus pour jouer un rôle dans le remodelage de la chromatine [12]. Le premier est composé d'enzymes capables de remodeler la chromatine de manière ATPdépendante $[37,45]$. Le second est impliqué dans des modifications post transcriptionnelles des extrémités des histones, comme l'acétylation ou la méthylation [5]. L'acétylation des résidus lysine des queues $\mathrm{N}$-terminales des histones cœur a été très étudiéc. Les enzymes impliquées dans la régulation de l'acétylation des histones sont les HAT (histones acétyl transférases) qui acétylent les histones au niveau de résidus lysine spécifiques et les HDAC (histone déacétylases) qui désacétylent les histones [27]. Il est maintenant établi que les modifications du degré d'acétylation des histones cœur sont impliquées dans la régulation de la transcription. De plus de nombreuses études ont montré que la chromatine acétylée était transcriptionnellement active alors que la chromatine hypoacétylée correspondait aux régions inactives du génome $[7,42]$. Dans plusicurs situations des facteurs de remodelage ATP. dépendants et des HATs ont été montrés comme étant impliqués simultanément dans la régulation de gènes spécifiques $[10,25]$. Cependant, à l'heure actuelle, le lien entre l'acétylation de la chromatine et son remodelage n'est pas connu.

\section{Description du remodelage de la chromatine au cours de la spermatogenèse}

La spermatogenèse est la différenciation des cellules ger- minales depuis les spermatogonies jusqu'aux spermatozoïdes. Les spermatogonies entrent en méiose et deviennent ainsi les spermatocytes préleptotènes, lesquels dupliquent leur ADN et ensuite traversent les étapes leptotène, zygotène, pachytène et diplotène de la prophase de première division méiotique. Dans les spermatocytes au stade pachytène, les chromosomes homologues s'apparient et échangent du matériel chromosomique au cours du processus de recombinaison méiotique. Un grand nombre de protéines participent à ce processus, localisées dans des structures appelées complexes synaptonémaux (CSN) le long des chromosomes apparićs, au niveau des sites de recombinaison. A l'issue de la première division de méiose sont produits les spermatocytes II, lesquels traversent rapidement la deuxième division méiotique pour former les spermatides haploïdes [19].

Au cours de la maturation post-méiotique de la spermatide, ou spermiogenèse, un remodelage spectaculaire de la chromatine accompagne une mise au repos réversible de l'ensemble du génome. La transcription des gènes est active dans les spermatogonies, les spermatocytes au stade pachytène et dans les spermatides rondes. Elle cesse peu après dans les spermatides en élongation $[18,22,23,40$, 41]. Sur le plan morphologique, le noyau de la spermatide s'allonge puis se condense pour former le noyau spermatique, dont la chromatine présente une structure très spécifique et très compacte $[1,2,4,46,47]$. Lors de ce remodelage, les histones sont remplacées par les protéines de transition puis par les protamines $[11,38,39,51]$, protéines nucléaires spécifiques du spermatozoïde [3]. Or il a été montré dans plusieurs espèces qu'immédiatement avant leur remplacement, les protéines histones étaient hyperacétylées, notamment chez la truite [8], le coq [35] et le rat $[14,15,29]$. Au cours de la spermiogenèse che $z$ l'homme, où $85 \%$ des histones sont remplacées par les protamines, les histones restantes sont hautement acétylées dans les spermatozoïdes [13].

\section{Plusieurs études ont analysé le remplacement séquentiel des nucleoprotéines et/ou les change- ments structuraux au cours de la maturation post- méiotique des spermatides}

Des expériences de knock-out des protéines de transition et des protamines ainsi que l'étude séquentielle du remplacement des nucleoprotéines et des changements structuraux dans le noyau des spermatides en cours de maturation, ont tenté d'élucider les mécanismes de ces échanges et le rôle des différents acteurs. Bien que ces études donnent une vue plus précise de la séquence des évènements accompagnant le remodelage, elles n'apportent pas d'éclaircissement au sujet des mécanismes moléculaires et de la régulation de ce processus.

Par exemple, Kistler et al. [24] ont analysé l'expression 
séquentielle des protéines de transition et des protamines par électrophorèse sur gel de polyacrylamide de pools de tubules séminifères microdisséqués dont le stade a été identifié, ainsi que par immunohistochimie. Ils ont ainsi montré que chez le rat, TP2 est exprimé dans les spermatides aux stades 9-11 (bas niveau) et 12-14 (haut niveau), TPI dans les spermatides aux stades $12-15$ et Pl dans les spermatides à partir de la fin du stade 11 .

De même, Oko et al. [34], combinant la microscopie électronique et des immunodétections, ont décrit la réorganisation de la chromatine dans les spermatides de rat, lors de la disparition de l'histone spécifique de testicule, $\mathrm{H} 1 \mathrm{t}$ et l'apparition des protéines de transition TP1 et TP2. Ils ont notamment montré que, de manière contemporaine à la disparition de $\mathrm{Hlt}$, la chromatine du spermatide est reorganisée en un réseau fibrillaire, alors que l'apparition de TP1 et TP2 coïncide avec la condensation de cette chromatine fibrillaire dans une direction apico-caudale.

\section{L'étude des propriétés in vitro des protéines de} transition et des protamines ont conduit à plusieurs hypothèses concernant leur rôle au cours du remodelage chromatinien

Par exemple, Brewer et al. [6] ont récemment mesuré les taux de condensation et décondensation de l'ADN (ainsi que les constantes de dissociation correspondantes) induits par la fixation aux protéines de transition TP1 et TP2 et les protamines $\mathrm{P} 1$ et $\mathrm{P} 2$ du hamster Syrien. Ils ont ainsi montré que les deux protéines de transition condensent l'ADN libre à des taux similaires à ceux des protamines 1 et 2 et que les molécules d'ADN condensées par TP1 sont moins stables que celles condensées par les protamines ou TP2. Ils ont aussi observé que la portion correspondant aux 25 acides aminés C-terminaux de TP2 est responsable de la condensation de l'ADN.

\section{ACETYLATION DE LA CHROMATINE ET REMODELAGE CHROMATINIEN AU COURS DE LA SPERMIOGENESE CHEZ LES MAMMIFERES}

Malgré de nombreux efforts, la plupart de nos connaissances actuelles concernant les mécanismes impliqués dans le remodelage post-méiotique de la chromatine proviennent d'approches descriptives ainsi que de quelques expérimentations moléculaires menées in vitro. Parmi ces données, une attention particulière a été portée à l'acétylation des histones. En effet, une augmentation importante de l'acétylation globale des histones dans les spermatides a été observée dans de nombreuses espèces. Cet évènement précède immédiatement le remplacement des histones par les protéines de transition et les protamines.
Les mécanismes régissant le remplacement de la chromatine nucléosomale par les nucléoprotamines ne sont pas compris, mais il pourrait y avoir un lien entre l'acétylation globale des histones telle qu'elle est observée dans les spermatides en élongation et le remplacement des histones par les protamines dans les spermatides en condensation.

Les modifications post-traductionnelles des nucléoprotéines, incluant une acétylation extensive des queues $\mathrm{N}$-terminales des histones cœur, sont impliquées dans le remodelage de la chromatine au cours de la spermatogenèse.

Une hyperacétylation des histones a été observée dans les cellules post-méiotiques des testicules de nombreuses espèces animales, notamment chez la truite [8], le coq [35] et le rat $[14,15,29]$. En revanche, chez la carpe, où les histones somatiques sont entièrement retenues dans le spermatozoïde, $\mathrm{H} 4$ est non-acétylée dans les spermatides $[20$, 21].

En étudiant les profils de dénaturation de la chromatine dans des cellules germinales à plusieurs étapes de maturation, Grimes et Smart [16] ont observé une diminution importante de la stabilité thermique dans la chromatine des spermatides en élongation, laquelle pourrait résulter de l'hyperacétylation des histones et être associée à un relâchement de la chromatine dans ces cellules.

Dans le testicule de rat, une hyperacétylation de l'histone H4 a été observée in situ par Meistrich et al. [29], laquelle apparaît dans les spermatides en début d'élongation (stades 9-10) et devient maximum en fin d'élongation (stades 11 12). Dans ces derniers stades, le marquage le plus important a été observé dans la partie caudale des noyaux des spermatides.

Chez la souris [17] et chez l'homme (données non publiées), la détection des formes acétylées des histones cœur $\mathrm{H} 2 \mathrm{~A}, \mathrm{H} 2 \mathrm{~B}, \mathrm{H} 3$ et $\mathrm{H} 4$ par immunohistochimie sur coupes sériées de testicule a mis en évidence une hyperacétylation des histones cœur (Figure 1) dans les spermatides en cours d'élongation (types 8 à $11 \mathrm{chez}$ la souris, types 3 et 4 chez l'homme). Ces données ont été confirmées sur des Western blots réalisés à partir de populations cellulaires enrichies en spermatogonies, en spermatocytes, en spermatides rondes et allongées (ces populations de cellules testiculaires sont obtenues par sédimentation sur gradient de BSA). De plus une analyse topologique de la distribution nucléaire de l'histone $\mathrm{H} 4$ hyperacétylée par immunofluorescence et analyse d'images tridimensionnelle a mis en évidence une évolution chronologique de la distribution spatiale d'H4 hyperacétylée au sein du noyau de la spermatide en cours d'élongation [17] (Figure 2). D'après ces données, il apparaît que l'hyperacétylation de la chromatine précède immédiatement sa condensation, 


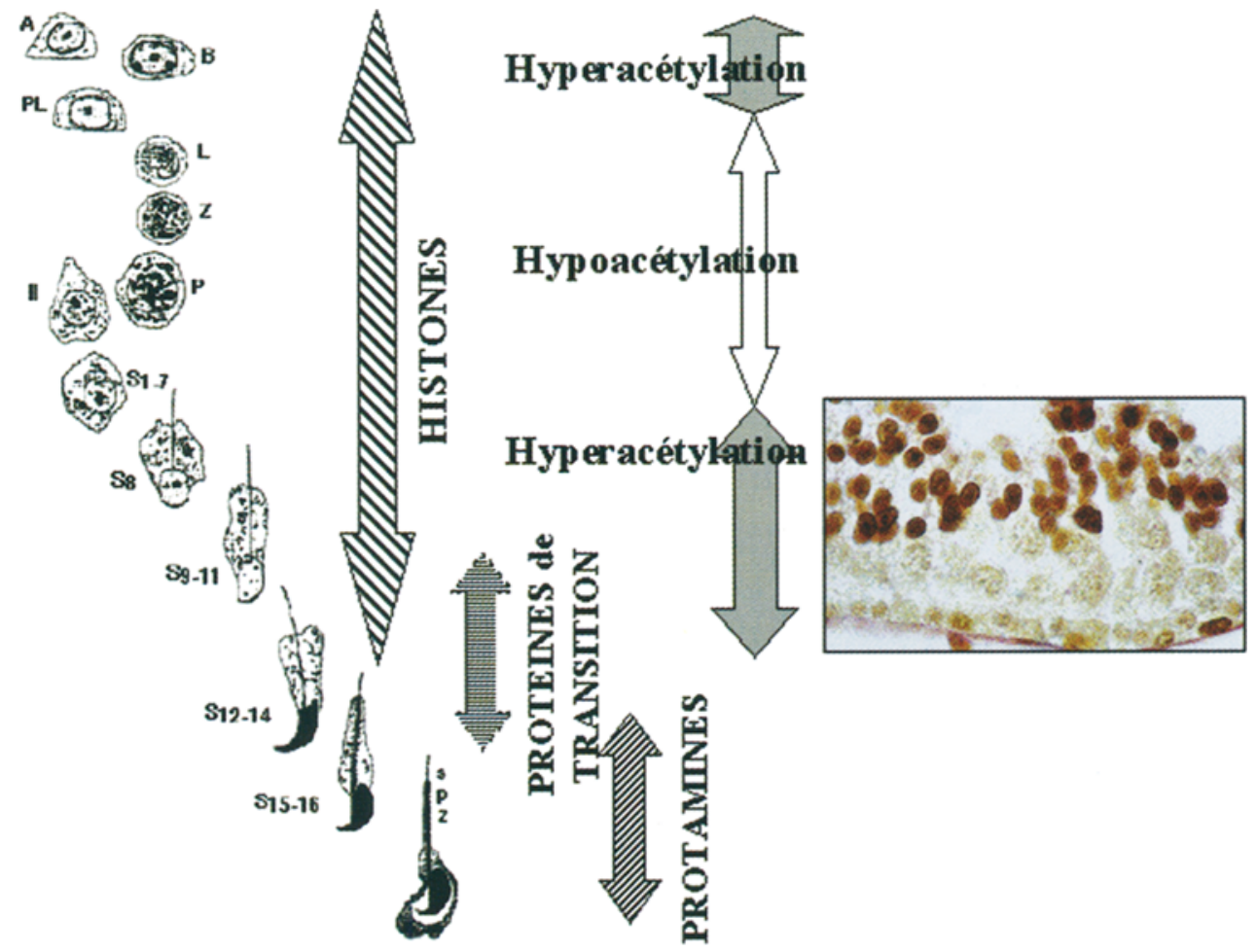

Figure 1 : Acétylation des histones au cours de la spermatogénèse murine.

$2 a$

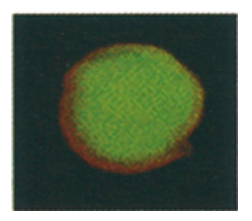

$2 \boldsymbol{b}$

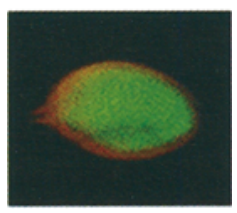

$2 c$

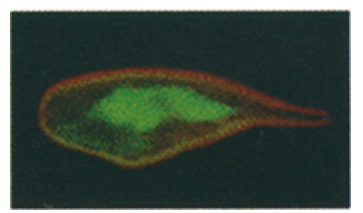

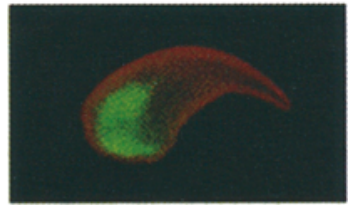

Figure 2 : Distribution spatiale de l'histone $\mathrm{H} 4$ acétylée dans les spermatides en cours d'élongation : aux stades précoces de l'élongation (2a et 2b), en milieu d'élongation (2c) et en fin d'élongation (2d). 
suggérant un rôle de signal permettant le recrutement et/ou l'activation des facteurs de remodelage.

Ainsi, chez la souris et chez l'homme, les histones cœur du nucléosome $\mathrm{H} 2 \mathrm{~A}, \mathrm{H} 2 \mathrm{~B}, \mathrm{H} 3$ et $\mathrm{H} 4$ sont acétylées dans les spermatogonies et les spermatocytes au stade préleptotène, déacétylées au cours de la méiose et dans les spermatides rondes, puis ré-acétylées dans les spermatides en élongation.

Les spermatogonies sont des cellules en cycle se divisant par mitose, et les spermatocytes préleptotènes sont des cellules qui subissent la réplication d'ADN précédant la méiose. Au cours de ces deux étapes, l'acétylation des histones observée pourrait correspondre au dépôt des nouvelles histones comme cela a été montré au cours de la phase S précoce chez Physarum par Loidl et Grobner [28] et lors de l'assemblage du nucléosome [36].

Lorsque les spermatocytes entrent en méiose, une diminution de l'acétylation est observée. Les histones cœur restent hypoacétylées pendant la longue prophase de méiose I, notamment au cours du stade pachytène, jusque dans les cellules post-méiotiques, les spermatides rondes. Ceci est surprenant de premier abord car les spermatocytes et les spermatides rondes sont connus pour être des cellules dont l'activité transcriptionnelle est importante $[9,31]$ et la transcription est associée à un haut niveau d'acétylation des histones [26]. Cependant, il est probable que l'hyperacétylation des histones cœur soit restreinte aux régions contenant les promoteurs des gènes activement transcrits dans ces cellules, et par conséquent soit en dessous de la limite de détection des méthodes utilisées ici, plus adéquates pour détecter une hyperacétylation globale du noyau. Cette hypothèse est confirmée par l'observation de Moens [30], qui a montré une accumulation de l'histone H4 dans les régions euchromatiques des spermatocytes au stade pachytène.

Pendant la maturation post-méiotique de la spermatide, il n'y a pas de réplication et la transcription s'arrête au début de la phase d'élongation [22, 23, 31]. Ainsi l'hyperacétylation des histones cœur dans la spermatide en élongation n'est liée ni à une réplication ni à la transcription. Il est probable qu'elle soit associée au remplacement des histones par les protéines de transition et les protamines. Pour étayer cette hypothèse, nous avons mis en évidence que la protéine TP2 était présente dans le spermatide en début de condensation au stade 11 alors que les histones acétylées sont encore présentes. Au stade 12, les histones acétylées ne sont plus détectables, mais la protéine de transition TP2 est toujours présente. Ceci montre que l'acétylation des histones précède et même se superpose au début du phénomène de remplacement des histones par les protéines de transition. De manière similaire, nos données 3D montrent que l'histone H4 acétylée dans la spermatide en cours d'é- longation est distribuée de manière spécifique. Dans la spermatide en tout début d'élongation (stade $8 \mathrm{chez}$ la souris), $\mathrm{H} 4$ acétylée est distribuée de manière homogène dans tout le noyau. Ensuite, aux stades 9 et 10, elle est localisée dans des régions plus restreintes du noyau et disparaît notamment de la région antérieure du noyau qui a commencé à se condenser. Enfin H4 acétylée se localise dans la région caudale de la spermatide en fin d'élongation, au stade 11 , région qui est la dernière à se condenser, puis disparaît complètement du noyau lorsque celui-ci est condensé.

L'expression des histones déacétylases est régulée au cours de la spermiogenèse. Elle est élevée dans les spermatocytes et dans les spermatides rondes, où elle est responsable d'un faible niveau d'acétylation des histones, puis décroit brutalement dans les spermatides en élongation.

Dans les cellules somatiques, le niveau d'acétylation des histones est le résultat de deux activités enzymatiques, les histone acétyl transferases (HATs) et les histone déacétylases (HDACs) [27]. Nous avons donc cherché à caractériser les activités HDAC et HAT au cours de la spermatogenèse et à identifier des facteurs impliqués dans la régulation de ces activités.

Le traitement de cellules germinales murines en suspension par un inhibiteur des histones déacétylases, la trichostatine A (TSA), induit une augmentation très importante de l'acétylation de la chromatine dans les spermatides rondes (dont la chromatine est normalement hypoacétylée) [17]. Ceci suggère que l'activité des histones déacétylases (HDAC) est responsable du maintien d'un faible niveau d'acétylation des histones dans les spermatides rondes.

Des Western blots sur cellules germinales enrichies aux différents stades de la spermatogenèse, ont montré que plusieurs HDACs (notamment les HDACs de classe I, HDAC1, 2 et 3) seraient présentes dans les spermatogonies, les spermatocytes et les spermatides rondes pour diminuer puis disparaître ensuite dans les spermatides en élongation/condensation et les spermatozoïdes (Figure 3 ). Ainsi, une dégradation des HDACs est contemporaine de l'augmentation globale du degré d'acétylation des histones dans les spermatides en élongation. Ceci suggère que l'augmentation du degré d'acétylation des histones dans les spermatides en élongation serait due à une baisse brutale de l'activité HDAC. En revanche, certaines HATs, dont Gcn5, apparaissent abondantes dans les spermatocytes au stade pachytène, dans les spermatides rondes, en élongation et en condensation, ce qui suggère que l'activité HAT serait maintenue lors de l'élongation des spermatides, et que la balance HAT-HDAC serait en faveur des HATs à ce stade. 


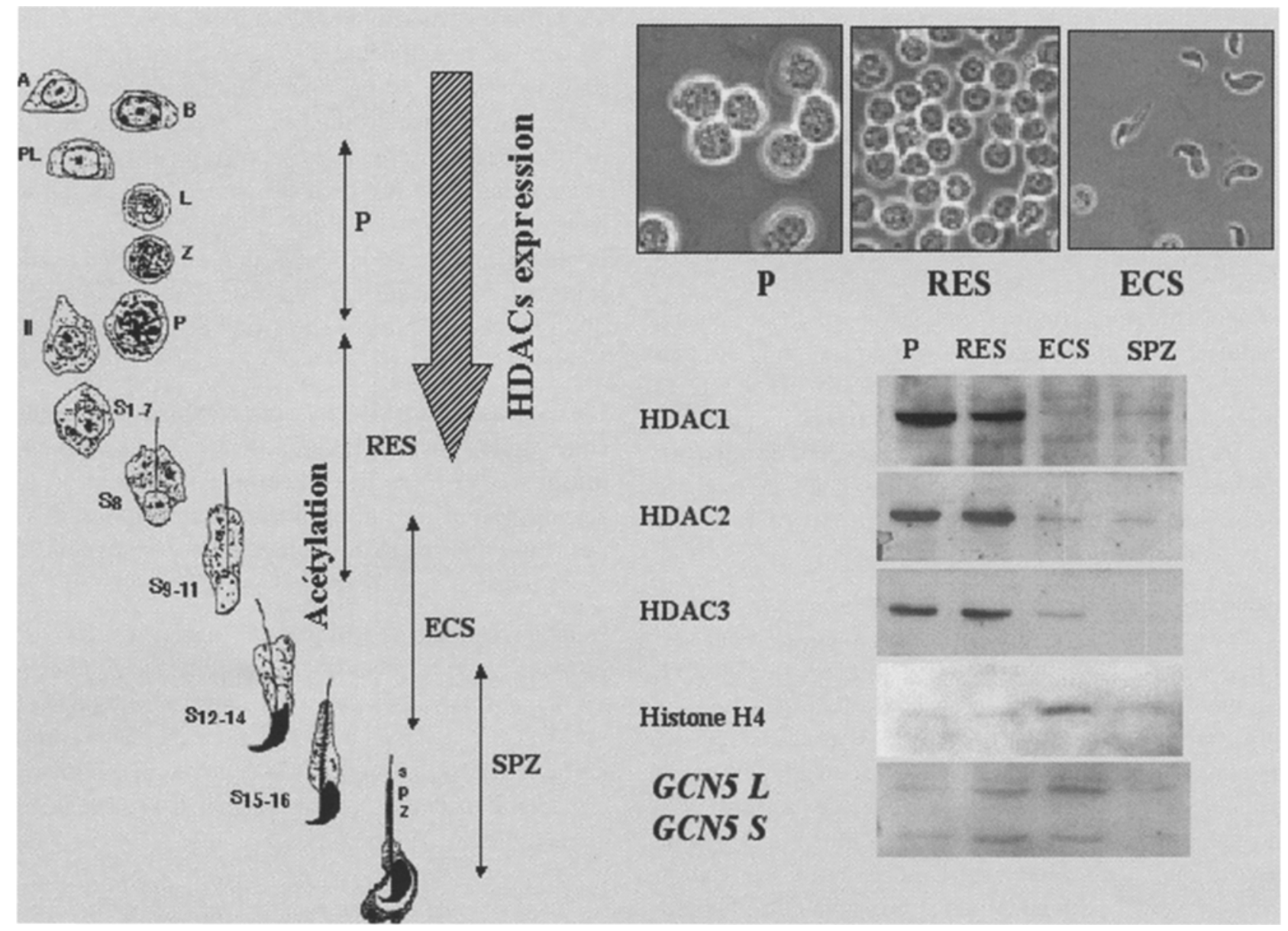

Figure 3 : Régulation de l'acétylation des histones au cours de la spermiogenèse : évolution de l'expression des HDACs et HATs.

$\boldsymbol{P}=$ spermatocytes au stade pachytène $; \mathrm{RES}=$ spermatides rondes et en élongation ; CES = spermatides en élongation et en condensation.

Chez l'homme, une absence de cellules germinales (SCO : Sertoli Cell Only Syndrome) est le plus souvent associée à une augmentation massive de l'acétylation des histones dans les cellules de Sertoli.

Sur des biopsies testiculaires humaines montrant une spermatogenèse normale ou subnormale, une hyperacétylation des histones est aussi mise en évidence dans les spermatides en début d'élongation. Ainsi, dans l'espèce humaine, des variations du niveau global d'acétylation des histones sont observées au cours des phases initiales de la spermiogenèse, selon un processus qui semble être conservé au cours de l'évolution. Les noyau des cellules de Sertoli montrent un niveau faible d'acétylation, le plus souvent non détectable par immunohistochimie (Figure 4).

Dans la plupart des situations où il existe une absence de cellules germinales dans les tubes séminifères, c'est à dire dans le syndrome des cellules de Sertoli isolées (Sertoli Cell Only ou SCO), une augmentation globale de l'acétylation des histones a été observée dans les noyaux des cellules de Sertoli (Figure 4). Celle-ci est observée, que le SCO soit associé ou non à une pathologie tumorale du testicule.

Cette observation suggère que le dialogue cellules germinales - cellules de Sertoli met en jeu des facteurs épigénétiques, et qu'une perturbation de ce dialogue est associée à des perturbations graves de la spermatogenèse, notamment dans les SCO et les cancers du testicule.

\section{CONCLUSION}

Ainsi, les histone cour $\mathrm{H} 2 \mathrm{~A}, \mathrm{H} 2 \mathrm{~B}, \mathrm{H3}$ et $\mathrm{H} 4$ sont hyperacétylées dans les spermatides en cours d'élongation. Ce changement d'acétylation est du à une dégradation des enzymes responsables de la déacétylation des histones, les histones déacétylases (HDACs) alors que les histones acétyl transférases (ou HAT) sont toujours présentes dans ces cellules. Il est hautement probable que cet évènement soit directement lié au remodelage global de la chromatine qui va suivre et au remplacement des histones par les protéines de transition et les protamines. Afin de décortiquer les mécanismes et les facteurs impliqués dans ce remodelage nucléaire, il reste à 


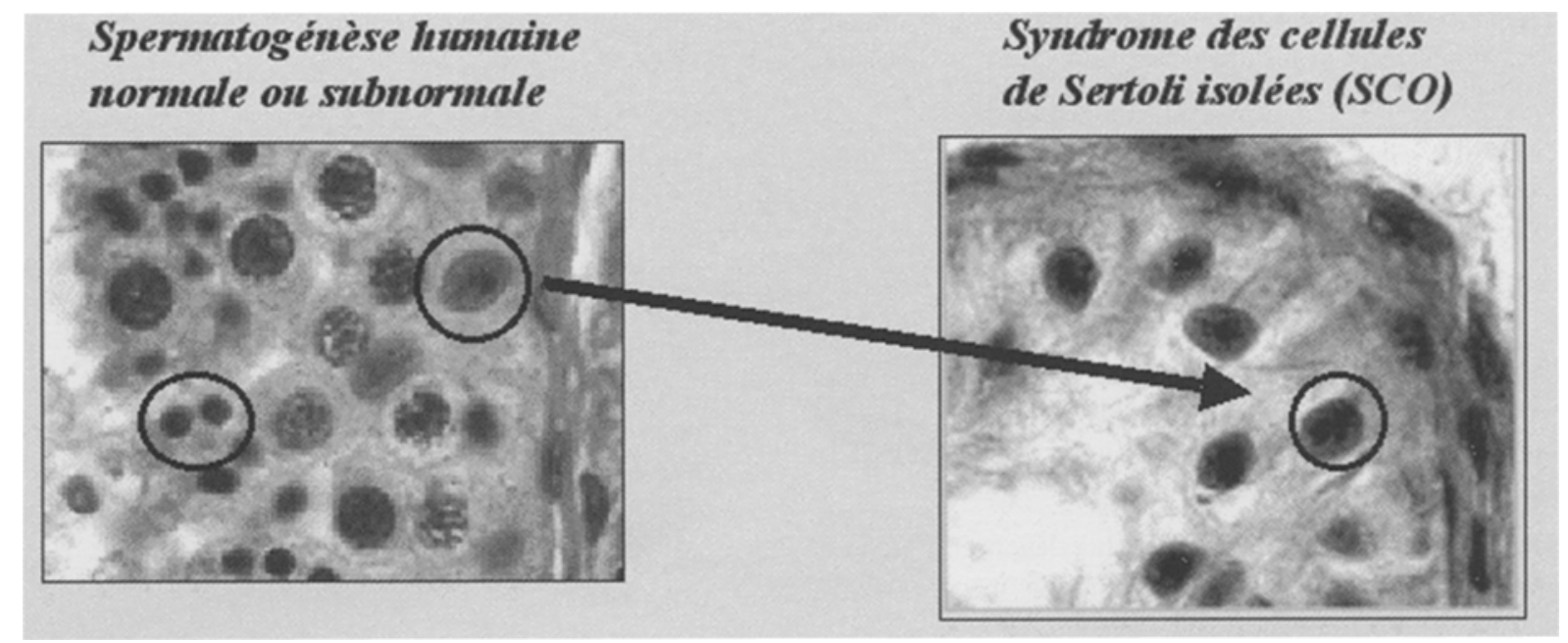

Figure 4 : Acétylation des histones au cours de la spermatogenèse humaine normale et lors du Syndrome des cellules de Sertoli isolées (SCO).

découvrir d'une part les facteurs responsables du changement d'équilibre HAT/HDAC, et d'autre part les facteurs potentiellement impliqués dans le remodelage de la chromatine acétylée. Ces derniers pourraient être «recrutés» par la chromatine acétylée, et induire des modifications structurales et/ou enzymatiques ellesmêmes responsables de l'échange de nucléoprotéines à la base de ce remodelage.

Enfin, lors d'une déplétion majeure des tubes seminifères en cellules germinales chez l'homme, il a été observé que les cellules de Sertoli, dont le noyau est normalement peu acétylé, subissent une augmentation globale de l'acétylation des histones dans leur noyau. Cette observation suggère que le remodelage de la chromatine au cours des étapes tardives de la spermatogenèse met en jeu un dialogue de nature «épigénétique» entre cellules de Sertoli et cellules germinales.

\section{REFERENCES}

1. BALHORN R., GLEDHILL B.L., WYROBEK A.J. : Mouse sperm chromatin proteins : quantitative isolation and partial characterization. Biochemistry, 1977, $16: 4074-4080$.

2. BALHORN R. : A model for the structure of chromatin in mammalian sperm. J. Cell Biol., 1982, $93:$ 298-305.

3. BALHORN R., WESTON S,, THOMAS C., WYROBEK A.J. : DNA packaging in mouse spermatids. Synthesis of protamine variants and four transition proteins. Exp. Cell Res., 1984, 150 298-308.

4. BALHORN R. : Mammalian protamines : structure and molecular interactions. In : Adolf K. eds. Molecular biology of chromsome function. New-York, Springer Verlag, 1989 : 366-395.

5. BERGER S.L. : Histone modifications in transcriptional regulation. Curr. Opin. Genet. Dev., 2002, 12 : 142-148.
6. BREWER L., CORZETT M., BALHORN R. : Condensation of DNA by spermatid basic nuclear proteins. J. Biol. Chem., 2002, $38: 895-900$.

7. CHEUNG W.L., BRIGGS S.D., ALLIS C.D. : Acetylation and chromosomal functions. Curr. Opin. Cell. Biol., 2000, 12 : 326333.

8. CHRISTENSEN M.E., DIXON G.H. : Hyperacetylation of histone $\mathrm{H} 4$ correlates with the terminal, transcriptionally inactive stages of spermatogenesis in rainbow trout. Dev. Biol., 1982, 93: 404-415

9. COOK P.R. : The transcriptional basis of chromosome pairing. J. Cell Sci., 1997, 110 : 1033-1040.

10. COSMA M.P., TANAKA T., NASMYTH K. : Ordered recruitment of transcription and chromatin remodeling factors to a cell cycle- and developmentally regulated promoter. Cell, 1999, 97 : 299-311.

11. DOENECKE D., DRABENT B., BODE C., et al. : Histone gene expression and chromatin structure during spermatogenesis. Adv. Exp. Med. Biol., 1997, 424 : 37-48.

12. FEATHERSTONE M. : Coactivators in transcription initiation : here are your orders. Curr. Opin. Genet. Dev., 2002, 12 : 149155 .

13. GATEWOOD J.M., COOK G.R., BALHORN R., SCHMID C.W., BRADBURY E.M. : Isolation of four core histones from human sperm chromatin representing a minor subset of somatic histones. J. Biol. Chem., 1990, 265 ; 20662-20666.

14. GRIMES S.R. Jr., HENDERSON N. : Hyperacetylation of histone H4 in rat testis spermatids. Exp. Cell Res., 1984, 152 : 91-97.

15. GRIMES S.R. Jr., HENDERSON N. : Acetylation of rat testis histones H2B and TH2B. Dev. Biol., 1984, 101 : 516-521.

16. GRIMES S.R. Jr., SMART P.G. : Changes in the structural organization of chromatin during spermatogenesis in the rat. Biochim. Biophys. Acta, 1985, 824 : 128-139.

17. HAZZOURI M., PIVOT-PAJOT C., FAURE A.K., et al. : Regu- 
lated hyperacetylation of core histones during mouse spermatogenesis: involvement of histone deacetylases. Eur. J. Cell Biol., 2000, 79 : 950-960.

18. HECHT N.B. : Molecular mechanisms of male germ cell differentiation. Bioessays, 1998, 20 : 555-561.

19. HESS R.A. : Spermatogenesis, overview. In : Knobil E. , Neill J.D. eds. Encyclopedia of Reproduction. San Diego, Academic Press, 1999.

20. KENNEDY B.P,. DAVIES P.L. : Acid-soluble nuclear proteins of the testis during spermatogenesis in the winter flounder. Loss of the high mobility group proteins. J. Biol. Chem., 1980, 255 : 2533-2539.

21. KENNEDY B.P., DAVIES P.L. : Phosphorylation of a group of high molecular weight basic nuclear proteins during spermatogenesis in the winter flounder. J. Biol. Chem., 1981, 256 : 92549259.

22. KIERSZENBAUM A.L., TRES L.L. : Structural and transcriptional features of the mouse spermatid genome. J. Cell Biol., $1975,65: 258-270$.

23. KIERSZENBAUM A.L., TRES L.L. : RNA transcription and chromatin structure during meiotic and postmeiotic stages of spermatogenesis. Fed. Proc., 1978, 37 : 2512-2516.

24. KISTLER W.S., HENRIKSEN K., MALI P., PARVINEN M. : Sequential expression of nucleoproteins during rat spermiogenesis. Exp. Cell Res., 1996, 225 : 374-381.

25. KREBS J.E., KUO M.H., ALLIS C.D., PETERSON C.L. : Cell cycle-regulated histone acetylation required for expression of the yeast HO gene. Genes. Dev., 1999, 13 : 1412-1421.

26. KUO M.H., BROWNELL J.E., SOBEL R.E., et al. : Transcription-linked acetylation by Gcn5p of histones $\mathrm{H} 3$ and $\mathrm{H} 4$ at specific lysines. Nature, 1996, $383: 269-272$.

27. KUO M.H., ALLIS C.D. : Roles of histone acetyltransferases and deacetylases in gene regulation. Bioessays, 1998, 20 : 615626.

28. LOIDL P., GROBNER P. : Postsynthetic acetylation of histones during the cell cycle : a general function for the displacement of histones during chromatin rearrangements. Nucleic Acids Res., 1987, $15: 8351-8366$.

29. MEISTRICH M.L., TROSTLE-WEIGE P.K., LIN R., BHATNAGAR Y.M., ALLIS C.D. : Highly acetylated H4 is associated with histone displacement in rat spermatids. Mol. Reprod. Dev., 1992, $31:$ 170-181.

30. MOENS P.B. : Histones $\mathrm{H} 1$ and $\mathrm{H} 4$ of surface-spread meiotic chromosomes. Chromosoma, 1995, 104 : 169-174.

31. NAYERNIA K., ADHAM I., KREMLING H. et al. : Stage and developmental specific gene expression during mammalian spermatogenesis. Int. J. Dev. Biol., 1996, 40 : 379-383.

32. OGURA A., YANAGIMACHI R. : Spermatids as male gametes. Reprod. Fertil. Dev., 1995, 7 : 155-158 ; discussion 158-159.

33. OGURA A., INOUE K., MATSUDA J. : Mouse spermatid nuclei can support full term development after premature chromosome condensation within mature oocytes. Hum. Reprod., 1999, 14 : 1294-1298.

34. OKO R.J., JANDO V., WAGNER C.L., KISTLER W.S., HERMO L.S. : Chromatin reorganization in rat spermatids during the disappearance of testis-specific histone, H1t, and the appearance of transition proteins TP1 and TP2. Biol. Reprod., 1996, $54: 1141-1157$.

35. OLIVA R., MEZQUITA C. : Histone $\mathrm{H} 4$ hyperacetylation and rapid turnover of its acetyl groups in transcriptionally inactive rooster testis spermatids. Nucleic Acids Res., 1982, 10 : 80498059.

36. PERRY C.A., DADD C.A., ALLIS C.D., ANNUNZIATO A.T. : Analysis of nucleosome assembly and histone exchange using antibodies specific for acetylated H4. Biochemistry, 1993, 32 : 13605-13614.

37. PETERSON C.L. : ATP-dependent chromatin remodeling: going mobile. FEBS Lett., 2000, 476 : 68-72.

38. POCCIA D. : Remodeling of nucleoproteins during gametogenesis, fertilization, and early development. Int. Rev. Cytol., 1986, $105: 1-65$.

39. RISLEY M.S. : Chromatin organization in sperm. London, CRC Press, 1990.

40. SASSONE-CORSI P. : Unique chromatin remodeling and transcriptional regulation in spermatogenesis. Science, 2002, 296 : 2176-2178.

41. SODERSTROM K.O., PARVINEN M. : RNA synthesis in different stages of rat seminiferous epithelial cycle. Mol. Cell. Endocrinol., 1976, 5 : 181-199.

42. STRAHL B.D., ALLIS C.D. : The language of covalent histone modifications. Nature, $2000,403: 41-45$.

43. TESARIK J., MENDOZA C., TESTART J. : Viable embryos from injection of round spermatids into oocytes [letter]. N. Engl. J. Med., 1995, 333 : 525.

44. VANDERZWALMEN P., NIJS M., STECHER A. et al. : Is there a future for spermatid injections ? Hum. Reprod., 1998, 13 : 7184.

45. VIGNALI M., HASSAN A.H., NEELY K.E., WORKMAN J.L. : ATP-dependent chromatin-remodeling complexes. Mol. Cell. Biol., 2000, 20 : 1899-1910.

46. WARD W.S. : The structure of the sleeping genome : implications of sperm DNA organization for somatic cells. J. Cell Biochem., 1994, $55:$ 77-82.

47. WARD W.S., ZALENSKY A.O. : The unique, complex organization of the transcriptionally silent sperm chromatin. Crit. Rev. Euk. Gene Expr., 1996, 6 : 139-147.

48. WARD W.S., KIMURA Y., YANAGIMACHI R. : An intact sperm nuclear matrix may be necessary for the mouse paternal genome to participate in embryonic development. Biol. Reprod., $1999,60: 702-706$.

49. WARD W.S., KISHIKAWA H., AKUTSU H., YANAGIMACHI H., YANAGIMACHI R. : Further evidence that sperm nuclear proteins are necessary for embryogenesis. Zygote, 2000, $8: 51$ 56.

50. WOLFFE A. : Chromatin- Structure and function. 2nd edition. Academic Press, London, 1995.

51. WOUTERS-TYROU D., MARTINAGE A., CHEVAILLIER P., SAUTIERE P. : Nuclear basic proteins in spermiogenesis. Biochimie, 1998, $80: 117-128$.

52. YANAGIMACHI R. : Production of mice through intracytoplasmic injection of sperm or spermatogenic cells. Protoplasma, 1999, 206 : 278-281. 


\section{ABSTRACT}

Factors involved in chromatin remodelling during spermatogenesis

Sophie ROUSSEAUX, Cécile CARON, Christophe PIVOT-PAJOT, Anne Karen FAURE, Mira HAZZOURI, Bernard SELE, Saadi KHOCHBIN

Round spermatids are post-meiotic cells with a haploid genome contained in a nucleus, with a structure initially similar to that of the somatic cell nucleus. During spermatogenesis, the spermatid nucleus undergoes drastic remodelling during which it first elongates and then condenses into the very specific and tightly packaged structure of the sperm nucleus. During this remodelling, the histones are replaced by transition proteins, which, in turn, are replaced by protamines, the specific nuclear proteins of the spermatozoa. Immediately prior to their replacement, the histones are hyperacetylated. The first part of our work was to precisely characterise the changes in histone acetylation during murine spermatogenesis. We have shown that the core histones $\mathrm{H} 2 \mathrm{~A}, \mathrm{H} 2 \mathrm{~B}, \mathrm{H} 3$ and $\mathrm{H} 4$ are hyperacetylated in the elongating spermatids. We have also shown that these changes in acetylation are associated with degradation of the enzymes responsible for histone deacetylation, histone deacetylases or HDACs, while histone acetyl transferases are still present in these cells. The histone acetylation pattern was also investigated during human spermatogenesis, revealing that histone hyperacetylation in the nucleus of elongating spermatids, which appears to be conserved during the course of evolution, also occurs during human spermatogenesis. Moreover, our data obtained from the testes of men with severely altered spermatogenesis, including SCO syndromes (Sertoli Cells Only Syndromes), show that a global hyperacetylation of the Sertoli cell nuclei is associated with an absence of meiotic and post-meiotic cells. This suggests that the global histone acetylation variations observed during spermatogenesis are part of a signalling pathway involving germ cell - Sertoli cell communication. Altogether, these data provide a basis for a better understanding of the mechanisms and identification of the factors involved in post-meiotic remodelling of chromatin.

Key-Words: chromatin, spermatogenesis, spermatid, histones, acetylation 